Research Article

\title{
Evaluation and Analysis of Traditional Physical Training by Using Mobile Edge Computing and Software-Defined Networking
}

\author{
Wenwen Pan $\mathbb{D}^{\mathrm{D}},{ }^{1}$ Jianzhi Wang $\mathbb{D}^{2},{ }^{2}$ and Jingsheng $\mathrm{Ji}^{1}$ \\ ${ }^{1}$ Qiqihar University, Qiqihar 161006, China \\ ${ }^{2}$ Mudanjiang Medical University, Mudanjiang 157011, China \\ Correspondence should be addressed to Jianzhi Wang; wangjianzhi@mdjmu.edu.cn
}

Received 11 January 2021; Revised 8 February 2021; Accepted 18 February 2021; Published 17 March 2021

Academic Editor: Jianhui Lv

Copyright (C) 2021 Wenwen Pan et al. This is an open access article distributed under the Creative Commons Attribution License, which permits unrestricted use, distribution, and reproduction in any medium, provided the original work is properly cited.

The body health plays an important metric in people's everyday life, and it directly determines whether people have the ability to preferably contribute to the society. In fact, the physical training is a universal sport to enhance the body health. Therefore, the evaluation and analysis of physical training become particularly significant. With the rapid development and emerging of new techniques and networking paradigms, the traditional offline physical training evaluation and analysis cannot be performed well. Instead, this paper uses Mobile Edge Computing (MEC) and Software-Defined Networking (SDN) to implement the evaluation and analysis of physical training, shortened for MSPT, where MEC is the new computing technique and SDN is the new networking paradigm. The proposed MSPT includes two parts. At first, the physical training data from different mobile devices are migrated into the edge server for computing according to the current condition, in which the game theory is used to complete the task scheduling. Then, SDN is responsible for the global scheduling in the centralized control manner, in which the multigranularity scheduling strategy is used to handle the traffic between the SDN controller and edge computing server. The experiments are driven by OMNet, including three aspects of evaluation, i.e., task offloading of MEC, traffic scheduling of SDN, and performance analysis of physical training, and the results show that the proposed MSPT has better performance than the corresponding baselines.

\section{Introduction}

The physical training has a huge following around the world and has been referred to as the national pastime since it can improve the body health greatly. According to a reliable survey, the number of persons which participate in the physical training program can reach $83.6 \%$ of the world's population [1], which further highlights that the physical training is an absolutely universal sport and plays an important role in people's everyday life. In spite of this, the improper physical training perhaps causes the accidental injury because the nonprofessional operations are usually adopted, especially for adolescents and elder population [2]. According to a rough report from the American Center for the Study of the Elderly, the proportion of which the old people are not obtained the proper guidance of physical training exceeds $28.9 \%$ [3]. Therefore, the correct and efficient conduction of physical training is very necessary. In other words, the evaluation and analysis of physical training become particularly significant.

However, it is far from enough to make the effective evaluation and analysis of physical training if only the traditional offline physical training way is used. Instead, it needs some new techniques or/and networking paradigms as the auxiliary tools to help complete the effective evaluation and analysis of physical training. As everyone knows, Mobile Edge Computing (MEC) $[4,5]$ is a new technique, and its purpose is to effectively solve problems such as latency and network load. In addition, MEC can put some complex tasks at the edge computing servers for computing, in order to save response time and thus guarantee the real-time evaluation and analysis. Analogically, in this paper, there is a lot of traffic related to evaluation and analysis of physical training, and this traffic can be regarded as those complex tasks. In particular, the current physical training usually uses the mobile devices to collect the training data. Thus, MEC 
can offload the collected data from these mobile devices into the edge computing servers for data computing.

In spite of this, the task offloading process of MEC can be covered, which has an important influence on the global evaluation and analysis of physical training. For this purpose, it needs to seek a networking paradigm to cover the whole network view. According to such demand, SoftwareDefined Networking (SDN) [6-8] is a very satisfactory candidate, which is a new future Internet networking paradigm with some special abilities. For example, SDN can grasp the global network view. According to such ability, the task offloading process of MEC can be taken in a glance. Besides, under SDN environment, all operations are performed in the centralized control manner; in other words, the evaluation and analysis of physical training are integrated.

Furthermore, as the introduction of SDN, it involves a necessary communication between the SDN controller and edge computing server, including traffic scheduling and message transmission (e.g., control signal). In order to improve the bandwidth utilization and save communication delay, the traditional object-level traffic scheduling $[9,10]$ between the SDN controller and edge computing server is discarded. Instead, this paper uses the multigranularity scheduling strategy to address different scenarios.

With the above consideration, this paper makes the evaluation and analysis of physical training by using MEC and SDN, called MSPT. The corresponding contributions are threefold. (1) In MEC, the game theory-based task offloading is performed. (2) In SDN, the multigranularity scheduling strategy between the SDN controller and edge computing server is devised. (3) The simulation experiments are implemented, including some main metrics' evaluation and analysis.

The rest paper is organized as follows. Section 2 reviews the related work, including task offloading in MEC and traffic scheduling in SDN. Section 3 presents the game theory-based task offloading. Section 4 devises the multigranularity scheduling strategy. Section 5 reports some major results. Section 6 concludes this paper.

\section{Related Work}

2.1. Task Offloading in MEC. There have lots of proposals on task offloading in MEC. In [11], there were three offloading options, i.e., nearest edge server, adjacent edge server, and remote cloud. It proposed a Reinforcement Learning (RL)based algorithm to make the optimal offloading decision for minimizing system cost, including energy consumption and computing time delay. In [12], a distributed many-tomany matching model was constructed to capture the interaction between mobile tasks and edge nodes, with the consideration of their diverse resource requirements and availabilities. It designed both distributed and centralized stable matching-based algorithms to jointly offload the tasks to edge nodes and determine their payments. In [13], a multiuser offloading scenario with intensive deployment of edge servers was considered. It divided the offloading process into two stages, i.e., data transmission and computation execution, in which the existence of Nash equilibrium was proven by using the noncooperative game method. In [14], the offloading decision problem was formulated as a 0-1 nonlinear integer programming problem under the constraints of channel interference threshold and the time deadline. Through the classification and priority determination for the mobile devices, a reverse auction-based offloading method was proposed to solve this optimization problem for energy efficiency improvement. In [15], the offloading problem was formulated as the joint optimization of computation task assignment and CPU frequency scaling, in order to minimize a tradeoff between task execution time and mobile energy consumption. It proposed a light-weight algorithm by using the Markov approximation technique to converge to a bounded near-optimal solution. In [16], a new task offloading scheme by considering the challenges of future edge, fog, and cloud computing paradigms was devised. To provide an effective solution toward an appropriate task offloading problem, it focused on two cooperative bargaining game solutions. The first method was used for timesensitive offloading services, and the second method was applied to ensure computation-oriented offloading services. In [17], the computation offloading framework was proposed by considering the quality of service, server resources, and channel interference, where the offloading decision was made based on the beneficial degree of computation offloading measured by the total cost of the local mobile devices. In [18], an intelligent computation offloading in combination with artificial intelligence technology was proposed. It devised a task migration algorithm based on task prediction according to the data size of computation task from mobile users and the performance features of edge computing nodes. In [19], the deep $\mathrm{RL}$ was proposed to solve the offloading problem of multiple service nodes for the cluster and multiple dependencies for mobile tasks in large-scale heterogeneous MEC. The proposed strategy had good performance in terms of energy consumption, load balancing, latency, and average execution time by using iFogSim and Google cluster trace. In [20], an optimal auction method for delay and energy constrained task offloading was proposed by a pair of deep neural networks. It maximized the profit of the edge servers while satisfied the task processing delay and energy consumption constraints of the mobile devices. In [21], a distributed task unloading strategy to the low load base station group under MEC environment was proposed, including two parts. At first, the communication resource, computing resource, and task queue of the low load base station group were modeled to quantify the energy cost in the process of task unloading. Then, the potential game model was used to solve the problem of distributed task unloading. In [22], the collaborative task offloading and data caching models were proposed to reduce the overall latency of all mobile devices. It used the Lyapunov online algorithm to perform the joint task offloading and dynamic data caching strategies for computation tasks or data contents. In [23], the data quality-aware offloading sequential decision making problem by adopting the 
principles of Optimal Stopping Theory (OST) was proposed to minimize the expected processing time. Besides, a variety of OST stochastic models and their applications to the offloading decision making problem were investigated.

2.2. Traffic Scheduling in $S D N$. There have also some proposals on traffic scheduling in SDN. For example, in [24], a nonsupervised deep learning-based routing strategy running in the SDN controller was proposed to address the impact on explosive growth in network traffic. In [25], a softwarized $5 \mathrm{G}$ architecture was first introduced for endto-end reliability of mission-critical traffic. Then, a mathematical framework was constructed to model the process of critical session transfers. Finally, a hardware implementation was conducted to study the practical effects of supporting mission-critical data at the core network level. In [26], an energy flow scheduling and routing mechanism in SDN was proposed to optimize the energy consumption both in link and switches, which could minimize traffic energy in time dimension and improve utilization of switches. In [27], a traffic engineering scheme based on reinforcement learning was proposed, which constructed and solved a simple linear programming problem to reroute the flows selected by reinforcement learning to balance utilization of link in the network. In [28], a multimedia traffic control mechanism based on deep reinforcement learning was proposed to achieve the multimedia traffic control and avoid the additional mathematical computation. In [29], an energysaving traffic scheduling algorithm was proposed to minimize the energy in hybrid software-defined wireless rechargeable sensor networks, which adequately used the features in SDN, such as direct control on SDN nodes and indirect control on normal nodes. In [30], a novel SDNbased architecture was devised to manage large-scale networks, which could guarantee quality of service and manage routing. The experiments showed that the proposed method could efficiently manage the hybrid architectures and reach good quality of service. In [31], the authors first formulated minimization time problem as an optimization problem and then proposed two polynomial-time algorithms to solve the optimization problem, which could test feasibility and obtain a solution, respectively. In [32], to minimize traffic load in softwaredefined wireless sensor networks, the authors first formulated the traffic load minimization problem as an optimization problem related to the optimization of relay sensor node and splitting flow transmission and then designed a Levenberg-Marquardt algorithm to solve the problem. In [33], a path determination and traffic scheduling strategy for SDN was proposed to ensure the bandwidth of quality of service to support businesses in network, which was able to obtain the optimal path with the port queues on switches.

Different from the abovementioned research studies, this paper uses the game theory to address the task offloading in MEC. In addition, this paper also designs the multigranularity scheduling strategy in SDN.

\section{Task Offloading between Mobile Device and Edge Computing Server}

3.1. Mathematical Modeling. The task offloading in MEC needs to offload some complex tasks from the mobile devices into the edge computing server for computing through the wireless base station. Different from the traditional task offloading model, this paper uses SDN to manage all traffic. As depicted in Figure 1, the network model of MSPT consists of four roles, i.e., mobile device, wireless base station, edge computing server, and SDN controller. Among them, the path between mobile device and wireless base station supports the $5 \mathrm{G}$ communication, and that between wireless base station and edge computing server supports the optical fiber communication. Therefore, the proposed network model can achieve the quickly interactive response and guarantee the low delay.

The process of task offloading inevitably involves the physical training data transmission. Suppose that there are $M$ wireless channels between mobile device and wireless base station and there are $N$ mobile devices, and for the arbitrary mobile device $U_{n}$, it has a given offloading strategy, denoted by $a_{n}$. If $a_{n}=0$, it means that these tasks will be computed at the local mobile device; otherwise, these tasks in the corresponding mobile device will be migrated into the edge computing server for computing. Let $a^{*}$ denote the global offloading strategy with respect to all mobile devices, denoted by $a^{*}=\left\{a_{1}, a_{2}, \ldots, a_{N}\right\}$, and the upstream link's transmission rate regarding the physical training data is defined as follows:

$$
\begin{aligned}
r_{n}\left(a^{*}\right) & =K * \log _{2}\left(1+\frac{S_{n}}{N_{i}}\right) \\
S_{n} & =q_{n} * g_{n} \\
N_{i} & =\sigma+\sum_{i=1}^{N} q_{i} g_{i} .
\end{aligned}
$$

Among them, $q_{n}$ is the transmission ability, $g_{n}$ is the transmission gain, and $\sigma$ is the white noise.

In addition to the network model and communication model, the computing model also plays an important role. For each mobile device, it has the single computing task, denoted by $I_{n}=\left(B_{n}, D_{n}\right)$, where $B_{n}$ is the data size of $I_{n}$ and $D_{n}$ is the required number of CPU cycles to complete $I_{n}$. For all computing scenarios in MEC, two cases are considered, i.e., local computing and offloading computing.

At first, let $t_{n}^{l}$ and $e_{n}^{l}$ denote the consumed time and consumed energy, respectively, and they are defined as follows:

$$
\begin{aligned}
& t_{n}^{l}=\frac{D_{n}}{s_{n}^{l}}, \\
& e_{n}^{l}=\gamma_{n}^{l} * D_{n},
\end{aligned}
$$

where $s_{n}^{l}$ is the CPU clock frequency of $U_{n}$ and $\gamma_{n}^{l}$ is the power dissipation of each CPU clock frequency. Let $K_{n}^{l}$ 


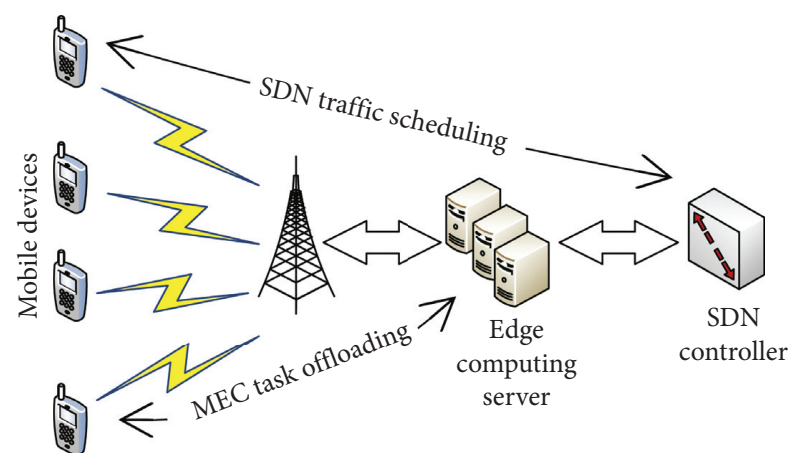

Figure 1: The network model of MSPT.

denote the total cost under the local computing case, and it is defined as follows:

$$
K_{n}^{l}=\lambda_{n}^{t} * t_{n}^{l}+\lambda_{n}^{e} * e_{n}^{l},
$$

where $\lambda_{n}^{t}$ and $\lambda_{n}^{e}$ are the weights regarding time consumption and energy consumption, respectively.

Then, let $t_{n \text {,tran }}^{c}\left(a^{*}\right)$ denote the upstream link's transmission time, $e_{n \text {,tran }}^{c}\left(a^{*}\right)$ denote the upstream link's energy consumption, $t_{n \text {,exe }}^{c}$ denote the required computing time, and $e_{n \text {,exe }}^{c}$ denote the required computing energy, respectively, and they are defined as follows:

$$
\begin{aligned}
t_{n, \text { tran }}^{c}\left(a^{*}\right) & =\frac{B_{n}}{r_{n}\left(a^{*}\right)}, \\
e_{n, \text { tran }}^{c}\left(a^{*}\right) & =\frac{q_{n} * B_{n}}{r_{n}\left(a^{*}\right)}, \\
t_{n, \text { exe }}^{c} & =\frac{D_{n}}{s_{n}^{c},} \\
e_{n, \text { exe }}^{c} & =\gamma_{n}^{c} * D_{n} .
\end{aligned}
$$

Among them, $s_{n}^{c}$ is the CPU clock frequency of edge computing server and $\gamma_{n}^{c}$ is the power dissipation of each CPU clock frequency. Here, this paper neglects two kinds of time/energy consumption, i.e., the path from edge computing server to mobile device, used to return the computing results, and the path from wireless base station to edge computing server. The first neglecting is because such path supports the optical fiber communication which has the ultra-low delay. The second neglecting is because the returned data size with respect to the physical training is very small, which takes nearly no delay. Let $K_{n}^{c}\left(a^{*}\right)$ denote the total cost under the offloading computing case, and it is defined as follows:

$$
K_{n}^{c}\left(a^{*}\right)=\lambda_{n}^{t}\left(t_{n, \text { tran }}^{c}\left(a^{*}\right)+t_{n, \text { exe }}^{c}\right)+\lambda_{n}^{e}\left(e_{n, \text { tran }}^{c}\left(a^{*}\right)+e_{n, \text { exe }}^{c}\right) .
$$

3.2. Game Theory-Based Optimization. For $a^{*}$, if most mobile devices select the offloading computing, these transmissions with respect to the physical training data perhaps cause the serious interference among them and thus generate some negative effects, such as increasing the system overhead including time consumption and energy consumption. Especially, when the total cost is larger than that under the local computing case, the offloading computing makes no sense. Therefore, in order to improve the overall revenue, the offloading computing has to be effective. Furthermore, for $a^{*}$, if the total cost under the offloading computing case is smaller than that under the local computing case, the corresponding edge computing server is effective. Mathematically,

$$
K_{n}^{c}\left(a^{*}\right)<K_{n}^{l} .
$$

For $N$ mobile devices, the optimization of edge computing belongs to NP-hard problem [34, 35]. Thus, this paper plans to use the game theory [36] to optimize the task offloading regarding $N$ mobile devices. Let $a_{N-n}$ denote the decision strategy set regarding all mobile devices excluding $U_{n}$, and it is defined as follows:

$$
a_{N-n}=\left(a_{1}, a_{2}, \ldots, a_{n-1}, a_{n+1}, \ldots, a_{N}\right) .
$$

Let $Z_{n}$ denote the current total cost, and the purpose of game theory is to minimize $Z_{n}\left(a_{n}, a_{N-n}\right)$. Mathematically,

$$
Z_{n}\left(a_{n}, a_{N-n}\right)= \begin{cases}K_{n}^{l}, & a_{n}=0, \\ K_{n}^{c}\left(a^{*}\right), & a_{n}>0 .\end{cases}
$$

Given $Z_{n}$, the task offloading optimization based on game theory is expressed as $\Gamma_{\mathrm{MEC}}=\left(N,\left\{a_{n}\right\},\left\{Z_{n}\right\}\right)$. Furthermore, let $a^{* *}=\left(a_{1}^{*}, a_{2}^{*}, \ldots, a_{N}^{*}\right)$ denote the global offloading strategy set when the Nash equilibrium is satisfied [13], and the corresponding mathematical express is shown as follows:

$$
Z_{n}\left(a_{n}^{*}, a_{N-n}^{*}\right) \leq Z_{n}\left(a_{n}, a_{N-n}^{*}\right) .
$$

According to the above statements, the game theorybased task offloading strategy in MEC is shown as the following five steps:

Step 1: $N$ mobile devices are initialized by considering that all tasks are completed at the corresponding mobile device.

Step 2: all wireless channels' transmission ability and transmission gain are computed.

Step 3: these wireless channels are arranged according to transmission ability and transmission gain in the descending order, and the wireless channel with the high power is assigned the high priority to make the offloading decision in advance.

Step 4: all offloading decision strategies and their corresponding total costs are computed. If equation (6) is satisfied, the current offloading decision strategy is unchanged. Otherwise, the current offloading decision strategy should be changed.

Step 5: the edge computing server sends the change request to the corresponding mobile device. If the corresponding mobile device receives the request, the offloading decision strategy is changed until all mobile 
devices are notified. Otherwise, the current offloading decision strategy is regarded as the optimal solution.

As the proposed network model supports $5 \mathrm{G}$ communication and optical fiber communication, the task offloading process usually has no the phenomenon of packet loss.

\section{Traffic Scheduling between Edge Computing Server and SDN Controller}

Section 3 addresses the task offloading between mobile device and edge computing server. As the task offloading process of MEC has an important influence on the global evaluation and analysis of physical training, it needs SDN to cover the whole network view. In other words, by using $\mathrm{SDN}$, the task offloading process of MEC can be taken in a glance. Therefore, this section pays attention to the traffic scheduling between the edge computing server and SDN controller. Furthermore, in order to improve the bandwidth utilization and save communication delay, this paper devises the multigranularity traffic scheduling strategy.

This paper considers two granularity change conditions, i.e., traffic aggregation and traffic splitting, which depends on the remaining bandwidth and the current network state. To be specific, if the current network state is smooth and there is enough network bandwidth, the traffic aggregation is approved. Otherwise, the traffic splitting is approved.

At first, for the coarse-grained traffic scheduling, the SDN controller periodically checks whether the network bandwidth satisfies to transmit the physical training data. When the traffic splitting condition is satisfied, the optimization purpose is to maximize the bandwidth utilization while minimize the number of core links. Mathematically,

$$
\text { minimize } \max \left\{U R_{l}\right\}, \quad l \in \mathrm{LC},
$$

where LC is the number of core links between the SDN controller and edge computing server.

The corresponding constraints are shown as follows:

$$
\begin{aligned}
U_{l}-\sum_{k=1}^{A_{l}} b w_{k}+\sum_{k=1}^{A_{l}}\left(\sum_{i=1}^{L} X_{P k i} * C_{l p k i}\right) * b w_{k} & =N_{l}, \quad N_{l}<T_{l}<t h r, \\
\sum_{i=1}^{P k} X_{P k i} & =1, \\
\sum_{k=1}^{A_{l}} \sum_{i=1}^{P k}\left(l p k i * X_{P k i} * C_{l p k i} * D_{l k j}\right) & <\text { delay }_{k}, \quad X_{P k i}, l p k i, C_{l p k i} \in\{0,1\} .
\end{aligned}
$$

Among them, $U_{l}$ is the used bandwidth link $l ; A_{l}$ is the aggregation traffic via link $l ; N_{l}$ is the bandwidth load of link $l ; X_{P k i}$ is the result of aggregation; $T_{l}$ is the total bandwidth of link $l$; $l p k i$ denotes the possibility of aggregation; $C_{l p k i}$ denotes whether the link $l$ is included; and $t h r$ is a given parameter to determine whether the congestion of link $l$ exits.

Then, for the fine-grained traffic scheduling, the SDN controller periodically checks the network bandwidth to schedule the physical training traffic from these congestion links to those light links as many as possible. Under this condition, the optimization purpose is defined as follows:

$$
\operatorname{minimize} \sum_{k=1}^{A_{l}} \sum_{i=1}^{P k}\left(l p k i * X_{P k i}\right) \text {. }
$$

It is obvious that the above two optimization objects belong to the 0-1 Integer Linear Programming (ILP) [37], and they can be solved easily. Thus, this paper gives the corresponding solution discussion no longer.

\section{Result Report}

The involved simulation parameters are set as follows: $N=10,20,30,40,50, \quad M=6, \quad B_{n}=26.5 \mathrm{~GB}, \quad D_{n}=1200 \mathrm{M}$ Cycle, $g_{n}=100 \mathrm{MW}$, and the network bandwidth of $5 \mathrm{G}$ communication is $1 \mathrm{Gbps}$. The hardware environment is shown as follows: CPU, Intel (R) Core (TM) i5-7400 CPU $4.68 \mathrm{GHz}$; RAM, 64 GB; Hard Disk, 1024 GB; OS, Windows 10 (64 bit). This section evaluates three aspects, including task offloading of MEC, traffic scheduling of SDN, and performance analysis of physical training. For the first part, the average time consumption and the average energy consumption are used as two evaluation metrics, where reference [19] is used as the baseline, called BMEC. For the second part, the average scheduling time and the average bandwidth utilization are used as two evaluation metrics, where reference [27] is used as the baseline, called BSDN. For the last part, the average response time and the average correct guidance rate are used as two evaluation metrics, where reference [38] is used as the baseline, called BPT.

5.1. Task Offloading Evaluation. For different mobile devices, the corresponding average time consumption with respect to MSPT and BMEC is shown in Figure 2. It is obvious that the average time consumption of MSPT is smaller than that of BMEC. In addition, with the increasing of mobile devices, the average time consumption becomes larger and larger, because more physical training data need to be handled. In particular, the average time consumption of MSPT shows the linear growth while that of BMEC shows 


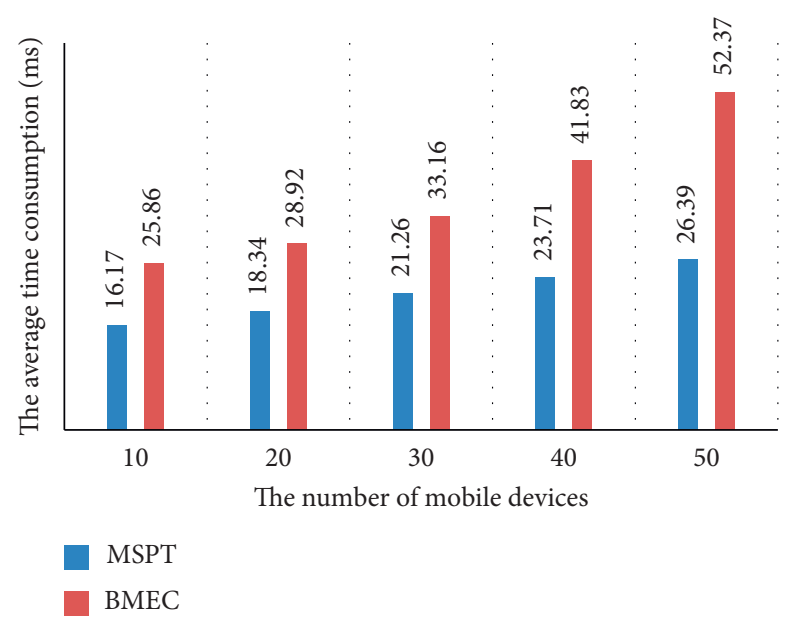

FIGURE 2: The average time consumption in case of task offloading evaluation.

the exponential growth, because BMEC uses the RL to solve the task offloading problem, which involves some iterations and generates more and more time consumption with the increasing of mobile devices.

For different mobile devices, the corresponding average energy consumption with respect to MSPT and BMEC is shown in Figure 3. At first, the average energy consumption of MSPT is smaller than that of BMEC, because MSPT uses the game theory to present a relatively optimal task offloading strategy and the corresponding energy consumption is limited. On the contrary, BMEC introduces the RL to repeatedly use the physical training data, which consumes too many energy inevitably. Then, similar to Figure 2, with the increasing of mobile devices, the corresponding average energy consumption increases. Besides, the increasing speed of MSPT is larger than that of BMEC.

5.2. Traffic Scheduling Evaluation. For MSPT and BSDN, the experimental results on the average scheduling time with respect to different mobile devices are shown in Figure 4. It is observed that MSPT has the significant advantage in terms of the average scheduling time, because it uses the multigranularity scheduling strategy to handle the physical training data. In addition, the devised multigranularity scheduling strategy in MSPT belongs to a heuristic algorithm, while the traffic scheduling strategy in BSDN is the $\mathrm{RL}$, which belongs to the intelligent algorithm. As a result, it needs too much time to handle the physical training data in terms of BSDN. Furthermore, with the increasing of mobile devices, the average scheduling time rises steadily, because the SDN controller needs to handle more physical training data.

For MSPT and BSDN, the experimental results on the average bandwidth utilization with respect to different mobile devices are shown in Figure 5. It can be found that MSPT has higher average bandwidth utilization than BSDN, because MSPT makes full use of the remaining network bandwidth according to the multigranularity scheduling, including two operations, i.e., traffic

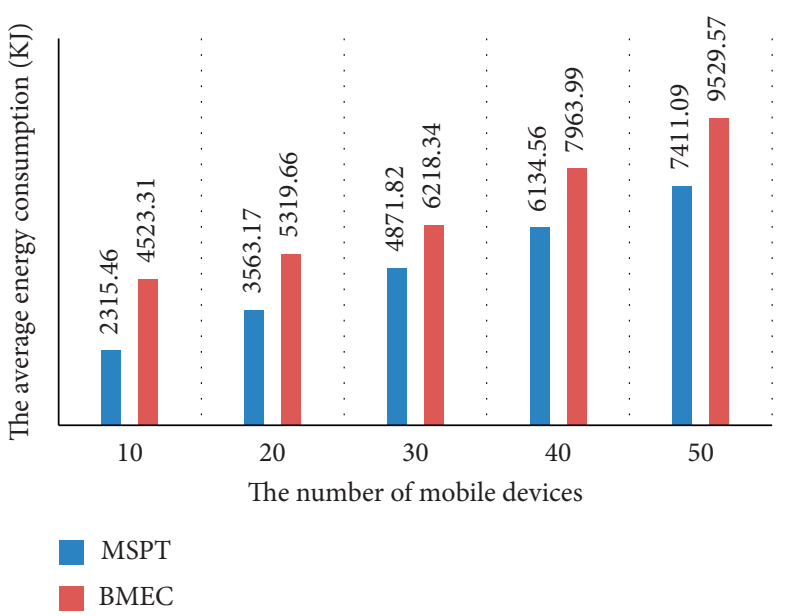

FIgURE 3: The average energy consumption in case of task offloading evaluation.

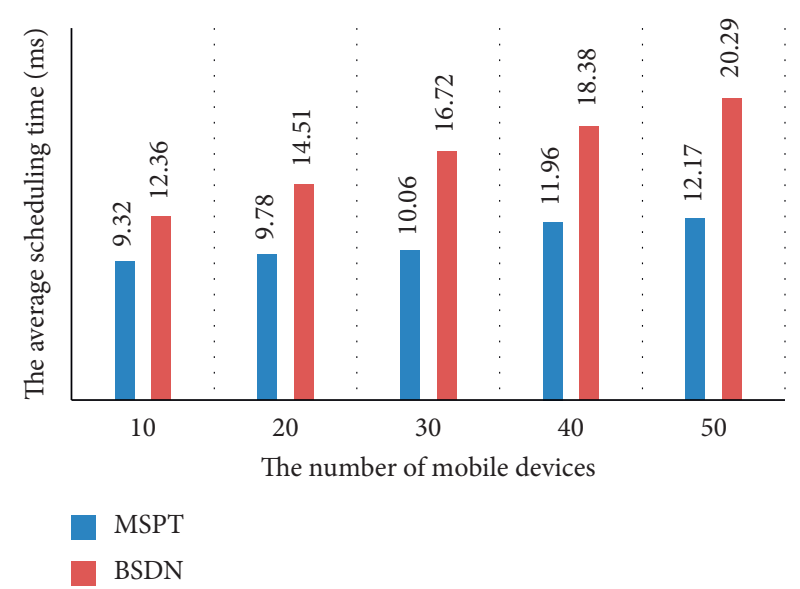

FIgURE 4: The average scheduling time in case of traffic scheduling evaluation.

aggregation and traffic splitting. If the current network bandwidth is sufficient, the traffic aggregation operation is adopted; otherwise, the traffic splitting operation is adopted. Unlike MSPT, BSDN only schedules the physical training data in the same granularity, which causes the congestion in terms of some links. Thus, the network bandwidth in BSDN cannot be leveraged timely.

5.3. Physical Training Evaluation. In this section, 1000 mobile devices and two edge computing servers are considered. For different simulation experiments, the corresponding average response time with respect to MSPT and BPT is shown in Table 1. It can see that the whole average response time of MSPT is smaller than that of BPT. It indicates that the game theory-based task offloading strategy and the multigranularity based traffic scheduling strategy have good optimization effect on the physical training. In particular, it only needs about $42 \mathrm{~ms}$ to obtain all physical training results in terms of these 1000 users, which is very satisfactory.

Furthermore, for different simulation experiments, the corresponding average correct guidance rate with respect to 


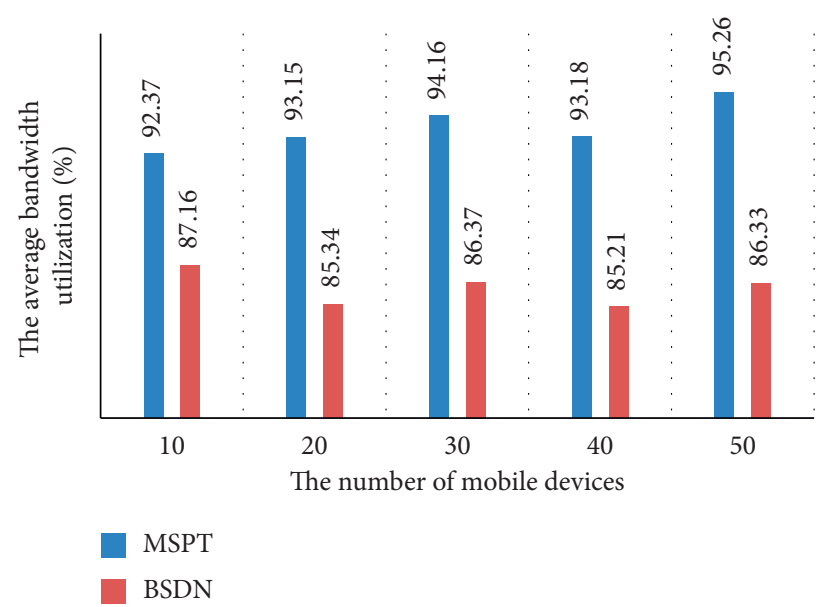

Figure 5: The average bandwidth utilization in case of traffic scheduling evaluation.

TABLE 1: The average response time in case of physical training evaluation $\backslash \mathrm{ms}$.

\begin{tabular}{lcccccccccc}
\hline No. & 1 & 2 & 3 & 4 & 5 & 6 & 7 & 8 & 9 & 10 \\
\hline MSPT & 42.18 & 43.56 & 42.39 & 42.57 & 42.66 & 43.05 & 43.11 & 42.83 & 42.91 & 42.16 \\
BPT & 61.33 & 62.09 & 62.85 & 61.94 & 62.53 & 62.74 & 61.95 & 62.17 & 63.52 & 62.82 \\
\hline
\end{tabular}

TABLE 2: The average correct guidance rate in case of physical training evaluation $\ \%$.

\begin{tabular}{lcccccccccc}
\hline No. & 1 & 2 & 3 & 4 & 5 & 6 & 7 & 8 & 9 & 10 \\
\hline MSPT & 100 & 100 & 100 & 100 & 100 & 100 & 100 & 100 & 100 & 100 \\
BPT & 98.34 & 97.95 & 98.26 & 96.98 & 97.18 & 98.23 & 97.87 & 98.06 & 98.16 & 97.46 \\
\hline
\end{tabular}

MSPT and BPT is shown in Table 2. It is obvious that the proposed MSPT has the absolutely higher average correct guidance rate than BPT. Especially, the correct guidance rate of MSPT always reaches $100 \%$, with a strong stability. As a result, SDN- and MEC-based physical training evaluation and analysis are very valuable.

\section{Conclusions}

The correct and efficient conduction of physical training plays an important role. In this paper, SDN and MEC are used to do the evaluation and analysis of physical training. At first, the task offloading in MEC is addressed, including the mathematical modeling (i.e., network model, communication model, and computing model) and the game theory-based offloading optimization strategy. Then, the traffic scheduling in SDN is addressed, where the multigranularity scheduling strategy is used to handle the traffic between the SDN controller and edge computing server, including traffic aggregation and traffic splitting.

For the proposed MSPT, three parts of experiments are performed: (1) the task offloading evaluation with time consumption and energy consumption consideration; (2) the traffic scheduling evaluation with scheduling time and bandwidth utilization consideration; (3) the physical training evaluation with response time and correct guidance rate consideration. In particular, the correct guidance rate of MSPT regarding the physical training can always reach $100 \%$.

\section{Data Availability}

The data used to support the findings of this study are available from the corresponding author upon request.

\section{Conflicts of Interest}

The authors declare that they have no conflicts of interest.

\section{Acknowledgments}

This work was supported by Philosophy and Social Science Research Planning Project in Heilongjiang Province (19TYC157), Research Project of Basic Scientific Research Operating Expenses of Provincial Institutions of Higher Learning in Heilongjiang Province (135409235), Heilongjiang Higher Education Teaching Reform Project (SYGY20200801), and University Nuring Program for Young Scholars with Creative Talents in Heilongjiang Porovince (UNPYSCT-2020077).

\section{References}

[1] N. E. Gilhus, "Physical training and exercise in myasthenia gravis," Neuromuscular Disorders, 2020, early view.

[2] Q. Lang, "Research on the integration of computer technology and physical education and training integrating computer algorithms," in Proceedings of the 2020 International Conference on Computer Information and Big Data Applications (CIBDA), pp. 440-445, Atlanta, GA, USA, December 2020. 
[3] J. S. Coom, A. Williams, and J. Radford, "Training health professionals to provide physical activity counselling," Progress in Cardiovascular Diseases, 2020, early view.

[4] A. Shakarami, M. Ghobaei-Arani, and A. Shahidinejad, "A survey on the computation offloading approaches in mobile edge computing: a machine learning-based perspective," Computer Networks, vol. 182, pp. 1-24, 2020.

[5] H. Elazhary, "Internet of Things (IoT), mobile cloud, cloudlet, mobile IoT, IoT cloud, fog, mobile edge, and edge emerging computing paradigms: disambiguation and research directions," Journal of Network and Computer Applications, vol. 128, pp. 105-140, 2019.

[6] L. Yang, B. Ng, W. K. G. Seah et al., "A survey on network forwarding in Software-Defined Networking," Journal of Network and Computer Applications, vol. 176, pp. 1-13, 2021.

[7] Y. Zhang, L. Cui, W. Wang et al., "A survey on software defined networking with multiple controllers," Journal of Network and Computer Applications, vol. 103, pp. 101-118, 2017.

[8] Y. Zhao, Y. Li, X. Zhang, G. Geng, W. Zhang, and Y. Sun, "A survey of networking applications applying the software defined networking concept based on machine learning," IEEE Access, vol. 7, pp. 95397-95417, 2019.

[9] K. Nisar, E. R. Jimson, M. H. A. Hijazi et al., "A survey on the architecture, application, and security of software defined networking: challenges and open issues," Internet of Things, vol. 12, pp. 1-27, 2020.

[10] Z. AlSaeed, I. Ahmad, and I. Hussain, "Multicasting in software defined networks: a comprehensive survey," Journal of Network and Computer Applications, vol. 104, pp. 61-67, 2017.

[11] T. Alfakih, M. M. Hassan, A. Gumaei, C. Savaglio, and G. Fortino, "Task offloading and resource allocation for mobile edge computing by deep reinforcement learning based on SARSA," IEEE Access, vol. 8, pp. 54074-54084, 2020.

[12] X. Wang, J. Wang, X. Zhang, X. Chen, and P. Zhou, "Joint task offloading and payment determination for mobile edge computing: a stable matching based approach," IEEE Transactions on Vehicular Technology, vol. 69, no. 10, pp. 12148-12161, 2020.

[13] S. Chen, Y. Chen, X. Chen et al., "Distributed task offloading game in multiserver mobile edge computing networks," Complexity, vol. 2020, Article ID 7016307, 14 pages, 2020.

[14] L. Li, X. Zhang, K. Liu et al., "An energy-aware task offloading mechanism in multiuser mobile-edge cloud computing," Mobile Information Systems, vol. 2018, Article ID 7646705, 13 pages, 2018.

[15] W. Zhou, W. Fang, Y. Li et al., "Markov approximation for task offloading and computation scaling in mobile edge computing," Mobile Information Systems, vol. 2019, Article ID 8172698, 12 pages, 2019.

[16] S. Kim, "New application task offloading algorithms for edge, fog, and cloud computing paradigms," Wireless Communications and Mobile Computing, vol. 2020, Article ID 8888074, 14 pages, 2020.

[17] N. Shan, Y. Li, and X. Cui, "A multilevel optimization framework for computation offloading in mobile edge computing," Mathematical Problems in Engineering, vol. 2020, Article ID 4124791, 17 pages, 2020.

[18] Y. Miao, G. Wu, M. Li et al., "Intelligent task prediction and computation offloading based on mobile-edge cloud computing," Future Generation Computer Systems, vol. 102, pp. 925-931, 2019.
[19] F. Lu, C. Gu, F. Luo et al., “Optimization of lightweight task offloading strategy for mobile edge computing based on deep reinforcement learning," Future Generation Computer Systems, vol. 102, pp. 847-861, 2019.

[20] F. Mashhadi, S. A. Salinas Monroy, A. Bozorgchenani et al., "Optimal auction for delay and energy constrained task offloading in mobile edge computing," Computer Networks, vol. 183, pp. 1-10, 2020.

[21] Y. Li and C. Jiang, "Distributed task offloading strategy to low load base stations in mobile edge computing environment," Computer Communications, vol. 164, pp. 240-248, 2020.

[22] N. Zhang, S. Guo, Y. Dong et al., "Joint task offloading and data caching in mobile edge computing networks," Computer Networks, vol. 182, pp. 1-10, 2020.

[23] I. Alghamdi, C. Anagnostopoulos, and D. P. Pezaros, "Data quality-aware task offloading in mobile edge computing: an optimal stopping theory approach," Future Generation Computer Systems, vol. 117, pp. 462-479, 2021.

[24] B. Mao, F. Tang, Z. M. Fadlullah et al., "A novel non-supervised deep-learning-based network traffic control method for software defined wireless networks," IEEE Wireless Communications, vol. 25, no. 4, pp. 74-81, 2018.

[25] V. Petrov, M. A. Lema, M. Gapeyenko et al., "Achieving endto-end reliability of mission-critical traffic in softwarized $5 \mathrm{G}$ networks," IEEE Journal on Selected Areas in Communications, vol. 36, no. 3, pp. 485-501, 2018.

[26] G. Xu, B. Dai, B. Huang, J. Yang, and S. Wen, "Bandwidthaware energy efficient flow scheduling with SDN in data center networks," Future Generation Computer Systems, vol. 68, pp. 163-174, 2017.

[27] J. Zhang, M. Ye, Z. Guo, C.-Y. Yen, and H. J. Chao, "CFR-RL: traffic engineering with reinforcement learning in SDN," IEEE Journal on Selected Areas in Communications, vol. 38, no. 10, pp. 2249-2259, 2020.

[28] X. Huang, T. Yuan, G. Qiao, and Y. Ren, "Deep reinforcement learning for multimedia traffic control in software defined networking," IEEE Network, vol. 32, no. 6, pp. 35-41, 2018.

[29] Y. Wei, X. Ma, N. Yang et al., "Energy-saving traffic scheduling in hybrid software defined wireless rechargeable sensor networks," Sensors, vol. 17, pp. 2126-2132, 2017.

[30] A. Bahnasse, F. E. Louhab, H. Ait Oulahyane, M. Talea, and A. Bakali, "Novel SDN architecture for smart MPLS traffic engineering-DiffServ aware management," Future Generation Computer Systems, vol. 87, pp. 115-126, 2018.

[31] J. Zheng, G. Chen, S. Schmid, H. Dai, J. Wu, and Q. Ni, "Scheduling congestion- and loop-free network update in timed SDNs," IEEE Journal on Selected Areas in Communications, vol. 35, no. 11, pp. 2542-2552, 2017.

[32] G. Li, S. Guo, Y. Yang et al., "Traffic load minimization in software defined wireless sensor networks," IEEE Internet of Things Journal, vol. 14, pp. 1-9, 2018.

[33] J. Xiao, S. Chen, and M. Sui, "The strategy of path determination and traffic scheduling in private campus networks based on SDN," Peer-to-Peer Networking and Applications, vol. 12, no. 2, pp. 430-439, 2019.

[34] L. Ma, S. Cheng, and Y. Shi, "Enhancing learning efficiency of brain storm optimization via orthogonal learning design," IEEE Transactions on Systems, Man, and Cybernetics: Systems, 2020, in press.

[35] W. Shi, Q. Li, C. Wang et al., LEAP: Learning-Based Smart Edge with Caching and Prefetching for Adaptive Video StreamingIEEE/ACM IWQoS, Phoenix, AZ, USA, 2019. 
[36] X. Molinero and F. Riquelme, "Influence decision models: from cooperative game theory to social network analysis," Computer Science Review, vol. 39, pp. 1-6, 2021.

[37] E. Klotz and A. M. Newman, "Practical guidelines for solving difficult mixed integer linear programs," Surveys in Operations Research and Management Science, vol. 18, pp. 18-32, 2016.

[38] L. Yuan and M. Wei, "Performance optimization mechanism of adolescent physical training based on reinforcement learning and markov model," Mobile Information Systems, vol. 2020, Article ID 8868225, 10 pages, 2020. 